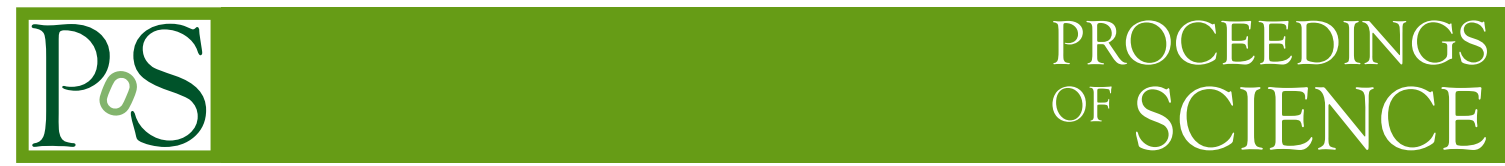

\title{
How well do we know the Unitarity Triangle? An experimental review
}

\section{Gabriella Sciolla* ${ }^{* \dagger}$}

Massachusetts Institute of Technology, Department of Physics, Room 26-443, 77 Massachusetts Avenue, Cambridge MA 02139

E-mail: sciolla@mit.edu

In the past 10 years our knowledge of the parameters $\rho$ and $\eta$ of the Cabibbo-Kobayashi-Maskawa matrix has improved substantially. This article reviews the measurements that contributed to this advance, and discusses their implication in terms of understanding $C P$ violation in the Standard Model and beyond.

KAON International Conference

May 21-25 2007

Laboratori Nazionali di Frascati dell'INFN, Rome, Italy

\footnotetext{
${ }^{*}$ Speaker.

${ }^{\dagger}$ Representing the BABAR Collaboration.
} 


\section{Introduction to the Unitarity Triangle}

According to Kobayashi and Maskawa [1], CP violation in the Standard Model (SM) is due to a complex phase appearing in the quark mixing matrix, the Cabibbo-Kobayashi-Maskawa (CKM) matrix. Following Wolfenstein's notation [2], the CKM matrix can be expressed in terms of the four real parameters $\lambda, A, \rho$ and $\eta$ as

$$
V=\left(\begin{array}{ccc}
V_{u d} & V_{u s} & V_{u b} \\
V_{c d} & V_{c s} & V_{c b} \\
V_{t d} & V_{t s} & V_{t b}
\end{array}\right)=\left(\begin{array}{ccc}
1-\lambda^{2} / 2 & \lambda & A \lambda^{3}(\rho-i \eta) \\
-\lambda & 1-\lambda^{2} / 2 & A \lambda^{2} \\
A \lambda^{3}(1-\rho-i \eta) & -A \lambda^{2} & 1
\end{array}\right)+O\left(\lambda^{4}\right) .
$$

While the parameters $\lambda$ and $A$ have been precisely known for a long time, the parameters $\rho$ and $\eta$ were poorly measured until recently. The parameter $\eta$ is of particular interest, because if $\eta=0$ the Standard Model would not be able to explain CP violation. If the CKM matrix is unitary, then $V^{+} V=1$. This implies six unitarity conditions that relate the nine elements of the matrix. The condition that relates the first and third columns of the matrix can be written as

$$
\frac{V_{u d} V_{u b}^{*}}{V_{c d} V_{c b}^{*}}+\frac{V_{t d} V_{t b}^{*}}{V_{c d} V_{c b}^{*}}+1=0
$$

This equation represents a triangle in the complex $(\rho, \eta)$ plane. This triangle, knows as the Unitarity Triangle (UT), is depicted in figure 1.

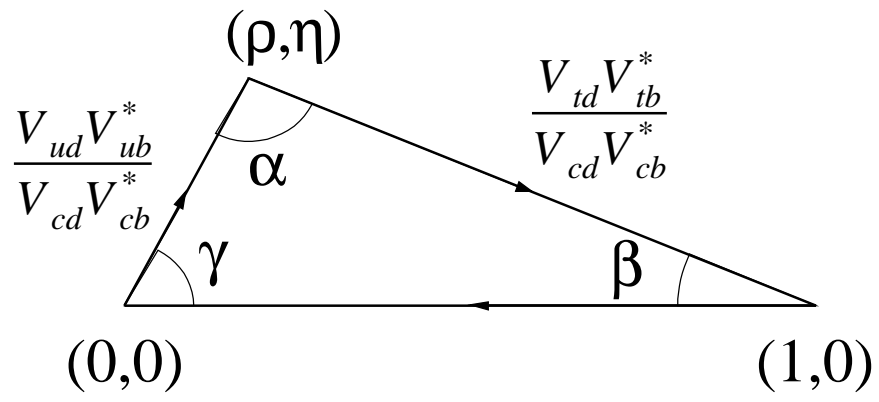

Figure 1: The Unitarity Triangle.

The study of $B$ and $K$ meson decays allows us to perform a number of measurements that set constraints in the $(\rho, \eta)$ plane. In the Standard Model all measurements must be consistent. The presence of New Physics could cause insconsistencies for some of the measurements of $\approx 10 \%$. A redundant and precise set of measurements providing constraints in the $(\rho, \eta)$ plane is therefore essential to test the CKM mechanisn and probe for New Physics beyond the Standard Model.

\section{The measurements}

The main contributors to this physics program are the two experiments at the asymmetric Bfactories, $B A B A R$ [3] and Belle [4]. Collectively, these experiments recorded to date over one billion $B \bar{B}$ pairs in $e^{+} e^{-}$interactions at the $\Upsilon(4 S)$ resonance. The large data set and clean experimental 
environment allowed the B factories to measure all sides and angles of the UT. The two Tevatron experiment, $\mathrm{CDF}$ and D0, add important constraints from their measurement of $B_{s}^{0}$ mixing. In addition, several kaon experiments (e.g., KTeV, NA48, KLOE) provide complementary information by measuring the $C P$-violating parameter $\varepsilon_{K}$ in $K^{0}$ decays.

\subsection{CP violation in $B^{0}$ decays}

The angles of the UT can be determined through the measurement of the time dependent $C P$ asymmetry, $A_{C P}(t)$. This quantity is defined as

$$
A_{C P}(t) \equiv \frac{N\left(\bar{B}^{0}(t) \rightarrow f_{C P}\right)-N\left(B^{0}(t) \rightarrow f_{C P}\right)}{N\left(\bar{B}^{0}(t) \rightarrow f_{C P}\right)+N\left(B^{0}(t) \rightarrow f_{C P}\right)},
$$

where $N\left(\bar{B}^{0}(t) \rightarrow f_{C P}\right)$ is the number of $\bar{B}^{0}$ that decay into the $C P$-eigenstate $f_{C P}$ after a time $t$.

In general, this asymmetry can be expressed as the sum of two components:

$$
A_{C P}(t)=S_{f} \sin (\Delta m t)-C_{f} \cos (\Delta m t)
$$

where $\Delta m$ is the difference in mass between $B^{0}$ mass eigenstates. The sine coefficient $S_{f}$ is related to an angle of the UT, while the cosine coefficient $C_{f}$ measures direct $C P$ violation.

When only one diagram contributes to the final state, the cosine term in equation 2.2 vanishes. As an example, for decays such as $B \rightarrow J / \psi K^{0}, S_{f}=-\eta_{f} \times \sin 2 \beta$, where $\eta_{f}$ is the $C P$ eigenvalue of the final state, negative for charmonium $+K_{S}$, and positive for charmonium $+K_{L}$. It follows that

$$
A_{C P}(t)=-\eta_{f} \sin 2 \beta \sin (\Delta m t)
$$

which shows how the angle $\beta$ is measured by the amplitude of the time dependent $C P$ asymmetry.

The measurement of $A_{C P}(t)$ utilizes decays of the $\Upsilon(4 S)$ into two neutral $B$ mesons, of which one $\left(B_{C P}\right)$ can be completely reconstructed into a $C P$ eigenstate, while the decay products of the other $\left(B_{\mathrm{tag}}\right)$ identify its flavor at decay time. The time $t$ between the two $B$ decays is determined by reconstructing the two $B$ decay vertices. The $C P$ asymmetry amplitudes are determined from an unbinned maximum likelihood fit to the time distributions separately for events tagged as $B^{0}$ and $\bar{B}^{0}$.

\subsection{The angle $\beta$}

The most precise measurement of the angle $\beta$ of the UT is obtained in the study of the decay $B^{0} \rightarrow$ charmonium $+K^{0}$. These decays, known as "golden modes," are dominated by a tree level diagram $b \rightarrow c \bar{c} s$ with internal $W$ boson emission (figure 2-a). The leading penguin diagram contribution to the final state has the same weak phase as the tree diagram, and the largest term with different weak phase is a penguin diagram contribution suppressed by $O\left(\lambda^{2}\right)$. This makes $C_{f}=0$ in equation 2.2 a very good approximation.

Besides the theoretical simplicity, these modes also offer experimental advantages because of their relatively large branching fractions $\left(\sim 10^{-4}\right)$ and the presence of narrow resonances in the final state, which provide a powerful rejection of combinatorial background.

The $C P$ eigenstates considered for this analysis are $J / \psi K_{S}, \psi(2 S) K_{S}, \chi_{c 1} K_{S}, \eta_{c} K_{S}$ and $J / \psi K_{L}$. 
a)

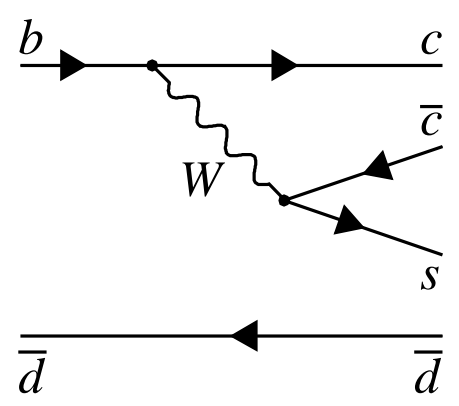

b)

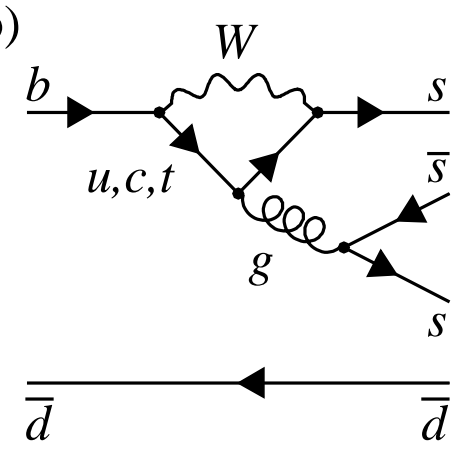

Figure 2: Feynman diagrams that mediate the $B^{0}$ decays used to measure the angle $\beta$ : a) $B^{0} \rightarrow$ charmonium $+K^{0} ;$ b) penguin dominated $B$ decays.

The asymmetry between the two $\Delta t$ distributions, clearly visible in figure 3 is a striking manifestation of $C P$ violation in the $B$ system. The same figures also display the corresponding raw $C P$ asymmetry with the projection of the unbinned maximum likelihood fit superimposed. The measurements from BABAR [5] and Belle [6] are averaged to obtain $\sin 2 \beta=0.678 \pm 0.026$ [7]. This measurement provides the strongest constraints in the $(\rho, \eta)$ plane.

An independent measurement of the angle $\beta$ through the study of B decays dominated by penguin diagrams allows us to search for physics beyond the Standard Model. In the SM, final states dominated by $b \rightarrow s \bar{s} s$ or $b \rightarrow s \bar{d} d$ decays offer a clean and independent way of measuring $\sin 2 \beta$ [8]. Examples of these final states are $\phi K^{0}, \eta^{\prime} K^{0}, f_{0} K^{0}, \pi^{0} K^{0}, \omega K^{0}, K^{+} K^{-} K_{S}$ and $K_{S} K_{S} K_{S}$. These decays are mediated by the gluonic penguin diagram illustrated in figure 2-b. In presence of physics beyond the Standard Model, new particles such as squarks and gluinos, could participate in the loop and affect the time dependent asymmetries [9].

A summary of the measurements of $A_{C P}(t)$ in penguin modes by the $B A B A R$ [10] and Belle [6] experiments is reported in figure 4 . The average of all the penguin modes, $0.56 \pm 0.05$ [7], is about $2 \sigma$ away from the value of $\sin 2 \beta$ measured in the golden mode.

\subsection{The angle $\alpha$}

If the decay $B^{0} \rightarrow \pi^{+} \pi^{-}$were dominated by the $b \rightarrow u$ tree level diagram, the amplitude of the time-dependent $\mathrm{CP}$ asymmetry in this channel would be a clean measurement of the parameter $\sin 2 \alpha$. Unfortunately, the analysis is complicated by substantial contributions from the gluonic $b \rightarrow d$ penguin amplitudes to this final state $(|P / T| \approx 30 \%)$. As a result, in the fit to the timedependent $\mathrm{CP}$ asymmetry (equation 2.2) one has to fit for both the sine and the cosine terms. The coefficient of the sine term is related to the angle $\alpha$ of the UT through isospin asymmetry.

A similar measurement can be performed using the decay $B^{0} \rightarrow \rho^{+} \rho^{-}$. This analysis is made difficult by the fact that since the $\rho$ is a vector meson, $\rho^{+} \rho^{-}$final states are characterized by three possible angular momentum states, and therefore they are expected to be an admixture of $C P=+1$ and $C P=-1$ states. However, polarization studies indicate that this final state is almost completely 

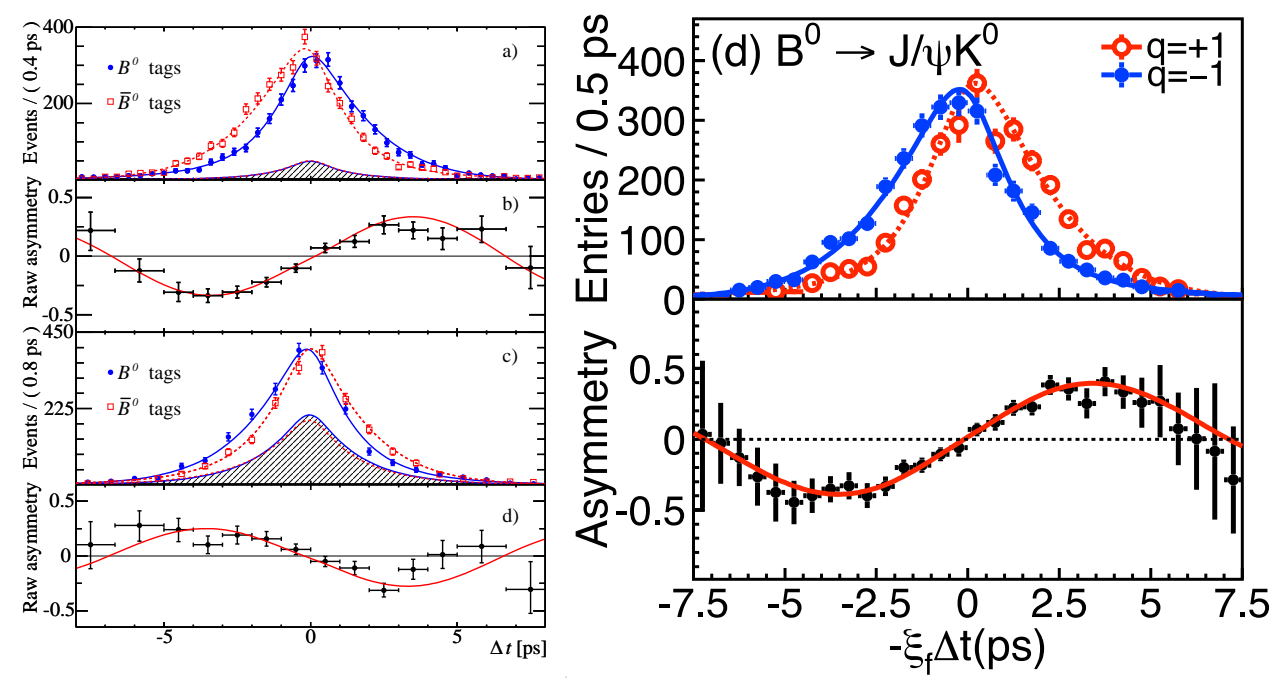

Figure 3: Measurements of $\sin 2 \beta$ in the "golden modes" by $B A B A R$ (left) and Belle (right). Left plot (BABAR): a) time distributions for events tagged as $B^{0}$ (full dots) or $\bar{B}^{0}$ (open squares) in $C P$ odd (charmonium $K_{S}$ ) final states; b) corresponding raw $C P$ asymmetry with the projection of the unbinned maximum likelihood fit superimposed; c) and d) corresponding distributions for $C P$ even $\left(J / \psi K_{L}\right)$ final states. Right plot (Belle): top) time distributions for events tagged as $B^{0}$ (open dots) or $\bar{B}^{0}$ (openfull dots) in charmonium $K_{S}$ final states; b) corresponding raw $C P$ asymmetry with the projection of the unbinned maximum likelihood fit superimposed.

longitudinally polarized, and therefore almost a pure $C P=+1$ eigenstate, which simplifies the analysis. Additional constraints are obtained by the study of $B \rightarrow \rho \pi$ decays.

Combining all BABAR and Belle results, we measure $\alpha=\left(92_{-9.3}^{+10.7}\right)^{\circ}[18]$.

\subsection{The angle $\gamma$}

The angle $\gamma$ is measured exploiting the interference between the decays $B^{-} \rightarrow D^{(*) 0} K^{(*)-}$ and $B^{-} \rightarrow \bar{D}^{(*) 0} K^{(*)-}$, where both $D^{0}$ and $\bar{D}^{0}$ decay to the same final state. This measurement can be performed in three different ways: utilizing decays of $D$ mesons to $C P$ eigenstates [11], utilizing doubly Cabibbo-suppressed decays of the $\bar{D}$ meson [12], and exploiting the interference pattern in the Dalitz plot of $D \rightarrow K_{S} \pi^{+} \pi^{-}$decays [13]. Currently, the last analysis provides the strongest constraint of the angle $\gamma$. Combining all results from BABAR and Belle, we measure $\gamma=\left(60_{-24}^{+38}\right)^{\circ}[18]$.

\subsubsection{The left side of the Unitarity Triangle}

The left side of the Unitarity Triangle is determined by the ratio of the CKM matrix elements $\left|V_{u b}\right|$ and $\left|V_{c b}\right|$. Both are measured in the study of semi-leptonic $B$ decays. The measurement of $\left|V_{c b}\right|$ is already very precise, with errors of the order of 1-2\% [7]. The determination of $\left|V_{u b}\right|$ is more challenging, mainly due to the large background coming from $b \rightarrow c \ell v$ decays, about 50 times more likely to occur than $b \rightarrow u \ell v$ transitions.

Two approaches, inclusive and exclusive, can be used to determine $\left|V_{u b}\right|$. In inclusive analyses of $B \rightarrow X_{u} \ell v$, the $b \rightarrow c \ell v$ background is suppressed by cutting on a number of kinematical 


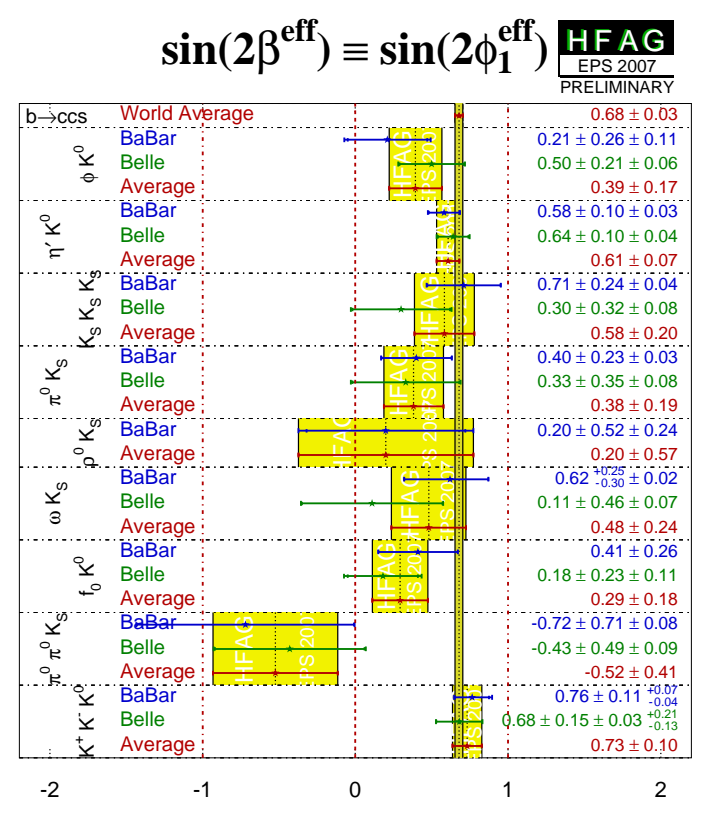

Figure 4: $B A B A R$ and Belle measurements of " $\sin 2 \beta$ " in the penguin dominated channels. The narrow yellow band indicates the world average of the charmonium $+K^{0}$ final states $\pm 1 \sigma$.

variables. This implies that only partial rates can be directly measured, and theoretical assumptions are used to infer the total rate and extract $\left|V_{u b}\right|$. The theoretical error associated with these measurements is $\approx 8 \%$. Averaging all inclusive measurements from the BABAR, Belle, and CLEO experiments we determine $\left|V_{u b}\right|=(4.31 \pm 0.17 \pm 0.35) \times 10^{-3}$ [7], where the first error is experimental and the second theoretical.

In exclusive analyses, $\left|V_{u b}\right|$ is extracted from the measurement of the branching fraction $B \rightarrow$ $\pi \ell v$. These analyses are usually characterized by a good signal/background ratio, but lead to measurements with larger statistical errors due to the the small branching fractions of the mode studied. In addition, the theoretical errors are also larger, due to the uncertainties in the form factor calculation. Both experimental and theoretical errors are expected to decrease in the future, making this approach competitive with the inclusive method.

\subsubsection{The right side of the Unitarity Triangle}

The right side of the Unitarity Triangle is determined by the ratio of the CKM matrix elements $\left|V_{t d}\right|$ and $\left|V_{t s}\right|$. This ratio can be determined with small $(\approx 4 \%)$ theoretical uncertainly from the measurement of ratio of the $B_{d}^{0}$ and $B_{s}^{0}$ mixing frequencies. The $B_{d}^{0}$ mixing parameter $\Delta m_{d}$ has been measured very precisely by many experiments [7]. The $B_{s}^{0}$ mixing parameter $\Delta m_{s}$, which escaped detection for many years due to the difficulty in detecting its very fast oscillations, was recently measured by the Tevatron experiments $[14,15]$. At the Tevatron, the $B_{s}^{0}$ mesons are exclusively reconstructed in their hadronic or semileptonic decays. Their flavor at production time is inferred by tagging the flavor of the other $B$ hadron produced in the opposite hemisphere, or by looking at the sign of fragmentation kaons produced in the same hemisphere. The time between production 
and decay of the $B_{s}^{0}$ mesons is then determined from the measurements of the boost of the $B_{s}$ meson and the distance between the interaction point and the $B$ meson decay vertex.

The value of $\Delta m_{s}$ measured by CDF is $(17.77 \pm 0.10 \pm 0.07) \mathrm{ps}^{-1}$. Combining this measurement with the world average for $\Delta m_{d}$, one can extract $\left|V_{t d} / V_{t s}\right|=0.2060 \pm 0.0007(\exp )_{-0.0060}^{+0.0081}$ (theo).

An independent determination of $\left|V_{t d} / V_{t s}\right|$ can be obtained by the measuring the ratio of the branching fractions $B F(B \rightarrow \rho \gamma) / B F\left(B \rightarrow K^{*} \gamma\right)$. Recent measurements of the braching fractions of $B F(B \rightarrow \rho \gamma)$ from BABAR [16] and Belle [17] yield $\left|V_{t d} / V_{t s}\right|=0.201 \pm 0.016(\exp ) \pm 0.015$ (theo).

The comparison between the two independent measurements of $\left|V_{t d} / V_{t s}\right|$, shown in figure 5, allows for an independent test of the Standard Model.

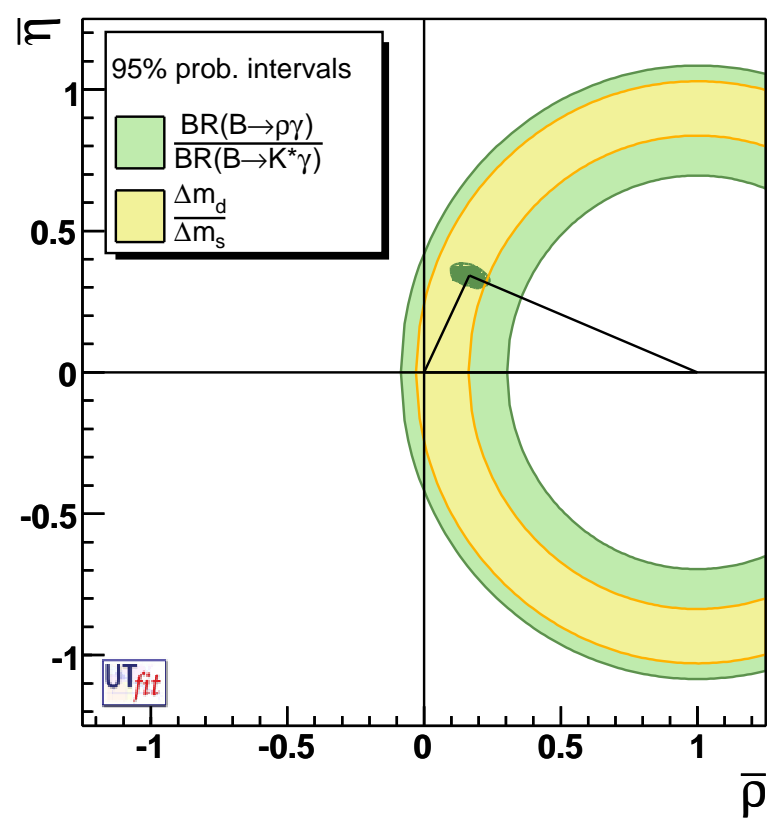

Figure 5: Comparison between the measurements of the right side of the UT from $B^{0}$ mixing (yellow band) and from $B F(B \rightarrow \rho \gamma) / B F\left(B \rightarrow K^{*} \gamma\right)$ (green band) from reference [19].

\subsubsection{Measurement of $\varepsilon_{K}$}

Kaon physics contributed the first constraint in the $(\rho, \eta)$ plane with the measurement of the parameter $\varepsilon_{K}$ in the study of $C P$ violation in neutral kaon decays. The parameter $\varepsilon_{K}$ is defined as

$$
\varepsilon_{K} \approx \frac{2}{3}\left|\eta_{+-}\right|+\frac{1}{3}\left|\eta_{00}\right|
$$

where

$$
\left|\eta_{+-}\right|=\frac{A\left(K_{L} \rightarrow \pi^{+} \pi^{-}\right)}{A\left(K_{S} \rightarrow \pi^{+} \pi^{-}\right)}=\sqrt{\frac{B F\left(K_{L} \rightarrow \pi^{+} \pi^{-}\right)}{\tau_{K_{L}}} \frac{\tau_{K_{S}}}{B F\left(K_{S} \rightarrow \pi^{+} \pi^{-}\right)}}
$$


and

$$
\left|\eta_{00}\right|=\frac{A\left(K_{L} \rightarrow \pi^{0} \pi^{0}\right)}{A\left(K_{S} \rightarrow \pi^{0} \pi^{0}\right)}=\sqrt{\frac{B F\left(K_{L} \rightarrow \pi^{0} \pi^{0}\right)}{\tau_{K_{L}}} \frac{\tau_{K_{S}}}{B F\left(K_{S} \rightarrow \pi^{0} \pi^{0}\right)}} .
$$

The 2006 PDG world average, including the latest measurements of the neutral kaon branching fractions from KTeV, NA48 and KLOE, is $\left|\varepsilon_{K}\right|=(2.232 \pm 0.007) \times 10^{-3}$ [20]. While the experimental accuracy on this measurement is remarkable $(0.3 \%)$, the corresponding constraints on the $(\rho, \eta)$ plane are not very stringent, mainly due to the theoretical uncertainties in the calculation of the bag parameter $B_{K}$ from Lattice QCD.

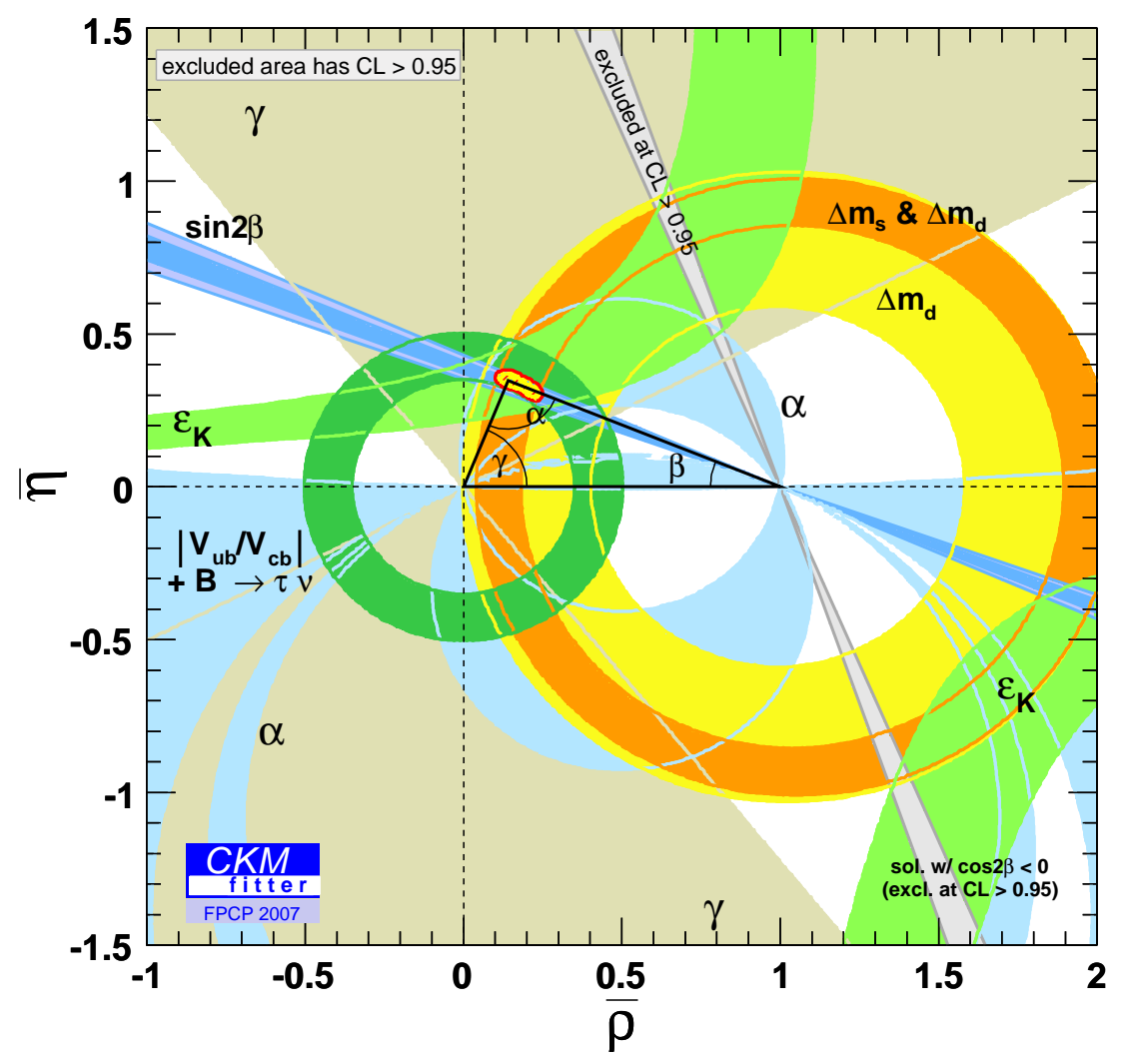

Figure 6: Constraints on the apex of the Unitarity Triangle resulting from all measurements.

\section{SUMMARY AND CONCLUSION}

Precise and redundant measurements of the sides and angles of the Unitarity Triangles provide a crucial test of $C P$ violation in the Standard Model. The constraints on the $(\rho, \eta)$ plane due to the measurements described in this article are illustrated in figure 6. The comparison shows good agreement between all measurements, as predicted by the CKM mechanism.

The accuracy of the mesurements is now approaching a few percent. This is the level of precision needed for detecting $\mathrm{O}(0.1)$ effects expected from New Physics. Additional data from 
the B factories, results from new-generation flavor experiments, and progress in theory especially lattice QCD, will be key to observing physics beyond the Standard Model in the flavor sector.

\section{References}

[1] M. Kobayashi and T. Maskawa, Prog. Theor. Phys. 49, 652 (1973).

[2] L. Wolfenstein, Phys. Rev. Lett. 51, 1945 (1983).

[3] B. Aubert et al. [BABAR Collaboration], Nucl. Instrum. Meth. A 479, 1 (2002).

[4] A. Abashian et al. [Belle Collaboration], Nucl. Instrum. Meth. A 479, 117 (2002).

[5] B. Aubert et al. [BABAR Collaboration], arXiv:hep-ex/0703021.

[6] K. F. Chen et al. [Belle Collaboration], Phys. Rev. Lett. 98, 031802 (2007).

[7] The Heavy Flavor Averaging Group, http://www.slac.stanford.edu/xorg/hfag/.

[8] D. London and R. D. Peccei, Phys. Lett. B 223, 257 (1989); N. G. Deshpande and J. Trampetic, Phys. Rev. D 41, 895 (1990); R. Fleischer, Z. Phys. C 62, 81 (1994); N. G. Deshpande and X. G. He, Phys. Lett. B 336, 471 (1994); Y. Grossman, Z. Ligeti, Y. Nir and H. Quinn, Phys. Rev. D 68, 015004 (2003); M. Gronau and J. L. Rosner, Phys. Lett. B 564, 90 (2003).

[9] A.B. Carter and A.I. Sanda, Phys. Rev. D 23, 1567 (1981); I.I. Bigi and A.I. Sanda, Nucl. Phys. B 193, 85 (1981); R. Fleischer and T. Mannel, Phys. Lett. B 511, 240 (2003);Y. Grossman, G. Isidori and M.P. Worah, Phys. Rev. D 58, 057504 (1998); Y. Grossman, Z. Ligeti, Y. Nir and H. Quinn, Phys. Rev. D 68, 015004 (2003); Y. Grossman and M.P. Worah, Phys. Lett. B 395, 241 (1997); R. Fleischer, Int. J. Mod. Phys. A 12, 2459 (1997); D. London and A. Soni, Phys. Lett. B 407, 61 (1997).

[10] B. Aubert et al. [BABAR Collaboration], arXiv:0706.3885 [hep-ex]; B. Aubert et al. [BABAR Collaboration], Phys. Rev. Lett. 98, 031801 (2007); B. Aubert et al. [BABAR Collaboration], hep-ex/0702046; B. Aubert et al. [BABAR Collaboration], arXiv:0706.3885 [hep-ex].

[11] M. Gronau and D. Wyler, Phys. Lett. B 265, 172 (1991); M. Gronau and D. London., Phys. Lett. B 253, 483 (1991).

[12] D. Atwood, I. Dunietz and A. Soni, Phys. Rev. Lett. 78, 3257 (1997).

[13] A. Giri, Y. Grossman, A. Soffer and J. Zupan, Phys. Rev. D 68, 054018 (2003).

[14] D0 Collaboration, D0note 5474-conf, presented at Lepton Photon 2007.

[15] Abulencia et al. [CDF Collaboration], Phys. Rev. Lett. 97, 242003 (2006).

[16] B. Aubert et al. [BABAR Collaboration], Phys. Rev. Lett. 98, 151802 (2007).

[17] Belle Collaboration, preliminary results presented at Lepton Photon 2007.

[18] The CKMfitter collaboration, http://ckmfitter.in2p3.fr/.

[19] The UTfit collaboration, http://utfit.roma1.infn.it/.

[20] W.-M. Yao et al. [Particle Data Group], J. Phys. G 33, 1 (2006). 